\title{
Simulations of the SIMBA array towards the determination of $\mathbf{Q}_{\beta}$ values
}

\author{
N. Warr ${ }^{1, a}$, A. Blazhev ${ }^{1}$, and K. Moschner ${ }^{1}$ \\ ${ }^{1}$ Institut für Kernphysik, Universität zu Köln, D-50937 Köln, Germany
}

\begin{abstract}
In an experiment at RIKEN, radioactive ions were implanted in the SIMBA silicon detector array and the $\beta$ particles from the subsequent decay detected. However, as SIMBA is not capable of complete calorimetry, it is not possible to extract $Q_{\beta}$ values directly from the data.

Geant4 simulations have been used to determine the response of SIMBA to a given $\beta$-emission spectrum. By simulating with different $\beta$ spectra and calculating the detector response to each spectrum, it is possible to compare with experiment and deduce the $Q_{\beta}$ values.

The technique is validated using two nuclei, for which the expected $\beta$ spectrum is known from the literature.
\end{abstract}

\section{The experiment}

The $\mathrm{N}=\mathrm{Z}$ nucleus ${ }^{94} \mathrm{Ag}$ was among those, in the ${ }^{100} \mathrm{Sn}$ region, studied via projectile fragmentation of a 345 $\mathrm{MeV} /$ nucleon ${ }^{124} \mathrm{Xe}$ beam on a ${ }^{9} \mathrm{Be}$ target using the EURICA array [1] at RIKEN. The nuclei of interest were separated by BigRIPS [2] and implanted in the Silicon IMplantation detector and Beta Absorber (SIMBA) array [3] (see fig. 1). The coincident $\gamma$ rays were detected by 12 Euroball clusters [4]. Several nuclei could be studied simultaneously and then selected by software gates. More details about the experiment can be found in [5].
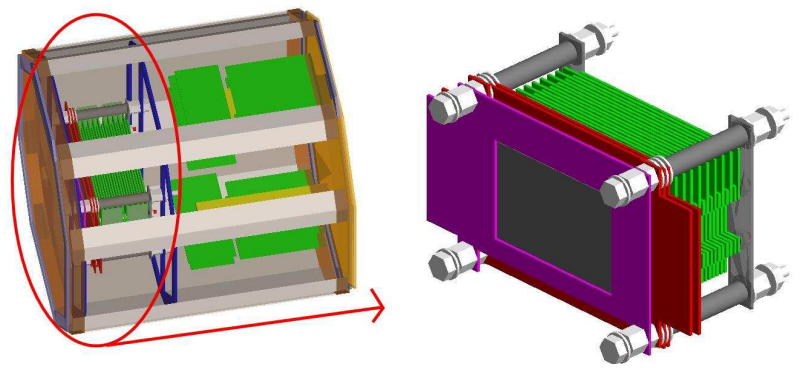

Figure 1. The SIMBA array. Left: with case, PCBs and support frame. Right: stack of Si detectors: XY detector (purple frame), implantation detector (red frames) and $\beta$ stack (green frames). The beam enters from the left in both cases and is stopped in the middle implantation detector.

The modified version of the SIMBA array (see fig. 1), which was used consisted of stacks of silicon detectors:

- A thin position-sensitive $(60 \times 40)$ double-sided siliconstrip XY detector (shown with a purple frame)

\footnotetext{
ae-mail: warr@ikp.uni-koeln.de
}

- A stack of three position-sensitive $(40 \times 60) 1-\mathrm{mm}$ thick implantation detectors (shown with red frames)

- A stack of twenty position-sensitive $(7 \times 1) 1-m m$ thick silicon detectors acting as a $\beta$ calorimeter (shown with green frames)

- Various support frames, printed-circuit boards (PCB) and the case

The combination of beam energy and detector thicknesses was chosen so that the ions were mostly stopped in the middle of the three implantation detectors.

Besides the use of SIMBA as a $\beta$ counter, another goal was to use it as a $\beta$-calorimeter to determine the $\mathrm{Q}_{\beta}$ values. The problem is that the $\beta$ particles scatter and may even have enough energy to pass through the entire stack of detectors. In this case, not all the energy of the $\beta$ particles is detected. Consequently, it is not possible to simply use the $\beta$ spectrum to determine the end-point energy, as this would underestimate the $\mathrm{Q}_{\beta}$ value.

Instead, it is necessary to determine the instrument response of SIMBA as a function of the $\beta$ spectrum. This means, that the $\beta$ spectrum is calculated from the end-point energy and used as an input to the simulation. All parts of SIMBA can scatter particles, so these must be tracked throughout. Monte-Carlo simulations built on the Geant4 [6] kernel were performed to this end, using a code written specifically for this purpose. In order to take advantage of multithreading, Geant4 version 10 was used.

This code allows the user to provide an initial $\beta$ spectrum and spatial distribution of the implantation area. For each event, Geant 4 randomly emits $\beta$ particles following this distribution and tracks them and all secondary particles as they scatter and are absorbed. The energy deposited in each active silicon volume is recorded for each event and can be analysed in the same manner as the experimental data. 


\section{The simulations}

The first step was to consider the response to monoenergetic positrons emitted from a nucleus implanted in the middle implantation detector. This showed that the setup can distinguish between different initial positron energies, according to which detector in the $\beta$ stack they are stopped in.

The next step was to simulate a real case, which could be compared to experimental data, namely ${ }^{98} \mathrm{Cd}$. This nucleus is known, from the literature, to decay to the $1691-\mathrm{keV}$ state in ${ }^{98} \mathrm{Ag}$, giving a $\beta^{+}$endpoint of 2707 $\mathrm{keV}$ [7]. Using this value, a $\beta^{+}$spectrum was generated (see left side of fig. 2) and used as input to the Geant4 simulation, which simulated the emission of this $\beta^{+}$ spectrum from the middle implantation detector (the beam energy having been chosen so as to stop the ions there). The Geant 4 code then calculated the scattering and detection of these $\beta$ particles and generated the simulated events for each detector. These events were then analysed in the same manner as the experimental data. By repeating with different values for the $\beta^{+}$endpoint, \pm 100 and \pm 500 $\mathrm{keV}$ either side of the literature value, it can be seen that the method is sensitive to a difference of $500 \mathrm{keV}$ but not to one of $100 \mathrm{keV}$.
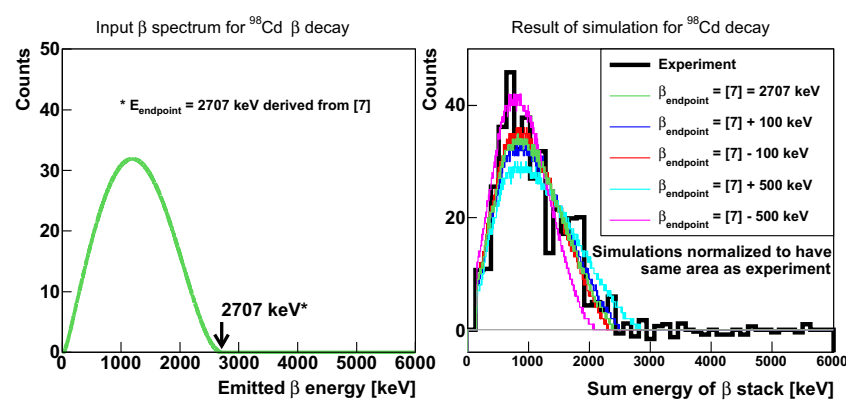

Figure 2. Left: input for the simulation following the implantation of ${ }^{98} \mathrm{Cd}$. Right: comparison between five simulations and the experimental data for ${ }^{98} \mathrm{Cd}$. It can be seen that the simulation based on the literature $\beta$ endpoint [7] (green) agrees with the data (black). It is not sensitive to a difference of $\pm 100 \mathrm{keV}$ (blue/red), but a difference of $\pm 500 \mathrm{keV}$ (cyan/magenta) from the literature value clearly disagrees with the experiment.

The second case of $\beta$ decay, which could be compared to the experimental data was ${ }^{96} \mathrm{Ag}$. In this case, the literature [8] indicates that most of the decay leads to the $2530-\mathrm{keV}$ state giving an endpoint at $8118 \mathrm{keV}$. However, Batist et al. showed that there is also a second branch to the 3184-keV state and a large fraction of $\beta$ feeding going to a continuum of excited states in ${ }^{96} \mathrm{Pd}$ around $7.5 \mathrm{MeV}$ [8].
The simulations show that if only the feeding of the $2530-\mathrm{keV}$ state is considered (see red curve of fig. 3), the experimental data are not well reproduced. However, if the other branches are included (blue curve of fig. 3) the simulation agrees better with the experiment (see in particular the $\beta$ sum energy in the range $1-1.5 \mathrm{MeV}$ ).

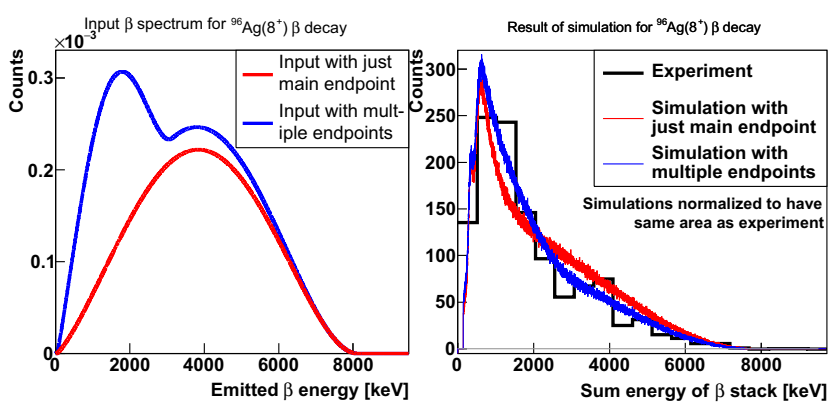

Figure 3. Left: inputs for the simulation following the implantation of ${ }^{96} \mathrm{Ag}$. Right: comparison between five simulations and the experimental data for ${ }^{96} \mathrm{Ag}$. If only the decay to the state at 2530 $\mathrm{keV}$ is considered (red curves), the agreement is poor. However, if the other branches observed by [8] are included (blue curves) the agreement is better.

\section{Conclusion}

The comparison between available experimental data and the simulations for known cases validate the simulation and indicates that the error is of the order of a couple of hundred $\mathrm{keV}$.

It is now possible to consider the cases of nuclei like ${ }^{94} \mathrm{Ag}$, where the $\beta$-endpoint energy is not yet known experimentally. Preliminary results indicate that this will be possible with the data from this experiment $[5,9]$.

\section{References}

[1] P.-A. Söderström et al., Nucl. Instr. Meth. B 317, 649 (2013)

[2] T. Kubo et al., Prog. Theor. Exp. Phys., 03C003 (2012)

[3] Ch. Hinke, PhD thesis, TU München (2010)

[4] J. Eberth et al. Prog. Part. Nucl. Phys. 28, 495 (1992)

[5] K. Moschner et al. contribution to these proceedings

[6] S. Agostinelli et al. Nucl. Instr. Methods A 506, 250 (2003)

[7] B. Singh and Z. Hu, Nucl. Data. Sheets 98, 335 (2003)

[8] L. Batist et al. Nucl. Phys. A 720, 245 (2003)

[9] K. Moschner et al. to be published 